\title{
Entrance-Surface-Dose Evaluation of Patients Examined by Diagnostic X-rays at Alhilal Alahmar Medical Center
}

\author{
Yousif Alghoul A* \\ Department of Physics, University of Sebha, Libya
}

*Corresponding author: Abubaker Ali Yousif Alghoul, Physics Department, Faculty of Science, University of Sebha, Libya, Email: abu.alghoul@sebhau.edu.ly

\section{Research Article}

Volume 5 Issue 2

Received Date: May 20, 2021

Published Date: August 20, 2021

DOI: $10.23880 /$ crij-16000188

\section{Abstract}

Medical Exposures from diagnostic X-rays machines are the most significant artificial source of human exposure to sparsely ionizing radiation. However there is lack of available data and relevant information on the evaluation of patient's entrance surface dose (ESD) from diagnostic X-Rays. In this research, the Entrance Surface Dose (ESD) is estimated for adult patients who have undertaken diagnosis X-ray examinations at Alhilal Alahmar Medical Center. The ESD has been estimated indirectly by using the X-Rays exposure factors for patients. The results of this research showed that the mean patient entrance surface doses (ESD) were 23.27 \pm 7.11 (mGy), 4.31 \pm 1.41 (mGy), 3.73 \pm 0.32 (mGy), 4.23 \pm 3.49 (mGy), $11.95 \pm 14.42$ (mGy) and $3.79 \pm 034$ (mGy) respectively for Lumbar Spine (LAT), Cervical Spine (LAT/AP), Chest (PA), Foot (AP/LAT), Knee (AP/LAT), and PNS (AP/ PA/LAT). The mean ESD values estimated are found to be slightly higher than the mean ESD reference values. This confirms the requirement for reducing the patients' doses to the tolerable levels recommended by many international radiation protection commissions.

Keywords: Patient Entrance Surface Dose (ESD); Diagnostic Medical X-rays

Abbreviations: ESD: Entrance Surface Dose; TLD: Thermoluminesent Dosimeters; IC: Ionization Chamber; FSD: Focus to Surface Distance; AP: Anterior Posterior; LAT: Lateral; PA: Posterior-Anterior.

\section{Introduction}

Medical examinations by diagnostic X-rays are an oldest recognized tool. All patients undergo these examinations benefit from them. However there are unavoidable effects from exposure to X-ray radiation. The ionization nature of the X-rays means that there is a risk of the exposure to this radiation. For example, one type of these effects is called deleterious effects, these effects occur when patients overexposure to these radiation. For this reason, applying radiation protection principles, in medical fields that using the X-rays procedures, is necessary. However, all exposures from diagnostic X-rays must be justified and optimized based on benefit and risk [1-3]. As it's well known as the $\mathrm{X}$-ray penetrates biological system it transfers a certain amount of energy, whereas this energy may be risky of unit cells building up these biological system of human body [4]. Internationally, there is increased concern about increasing level of patients exposing to diagnostic X-rays. This concern has been noticed in many published recommendations of the International Committee of Radiation Protection (ICRP) [5-7]. This concern is also observed in many other reports published by radiation protection organizations and in literature [8].

The almost recommendations are directed to oblige countries to provide restricted radiation dose to patients, whose undertaken X-ray examinations, in radiographic centers and, radiology departments those established at 


\section{Clinical Radiology \& Imaging Journal}

hospitals. One of the major significant factors that applied as an indicator of radiation protection, from deleterious effects, is patient dose. The patient dose specified usually by determining the entrance surface dose (ESD) for patients who exposed to diagnostic X-rays. The entrance surface dose (ESD) or skin entrance dose is generally defined as follows: The radiation absorbed dose by air at the point of intersection of X-ray beam axis with the entrance surface of the patient, which including backscatter radiation [9]. The entrance surface dose (ESD) is one of the basic quantities for measuring the patient dose and, as well for optimizing the given patient radiation dose. This quantity is basic criterion for comparing to the other international reference dose levels which is very significant from the point of view for radiation protection [10]. Many different methods for determining the ESD are established. In common, the ESD can be determined mainly by two techniques: Either by direct measurements of using Thermoluminesent dosimeters (TLD). These dosimeters are usually stacked on the patient's skin before X-ray exposure. The second way is indirectly through mathematical model calculations. These mathematical model calculations are based on the X-ray machine outputs. The ESD may as well be determined through measurements performed on a phantom beside the data from the patient's exploration [11-13].

Particularly, applying the TLDs in measuring the ESD for patients involves time consuming and, it is necessary to use special techniques which may not be available at the most radiographic centers. On the other hand, using ionization chambers to measure the ESDs for patients require applying conversion factors to change the ionization chamber (IC) reading to the absorbed dose. This procedure is a little bit difficult method [14]. Due to the hard accessibility of the TLD or the IC instruments, in addition to the lack of the calibration techniques of these instrumentations. To estimate the ESDs for patients the mathematical technique, which explained on the top of the text, is followed in this research. The aim of this research was to estimate the ESDs for patients exposed to diagnostic X-Rays at medical radiographic centers located in Sebha city.

\section{Materials and Methods}

This work was carried out in one of radiographic medical center located in city of Sebha. X-ray machine which used to expose patients is called Toshiba machine. The sample of adult patients exposed with this unit was 87 patients. The minimum inherent filtration in this unit is Aluminum equivalent $2 \mathrm{~mm} \mathrm{Al} / 75$, X-ray rating up to $150 \mathrm{kv}_{\mathrm{p}}$.

The X-Rays entrance surface dose (ESD) for each patient is estimated mathematically based on the X-Rays exposure factors. These factors are connected by using Chuan and Tsai formula [15]. This formula was applied to estimate ESD for many patients those exposed to diagnostic X-rays in six diagnostic examinations. Chuan and Tsai formula is given as follows:

Where $\mathrm{Q}$ is $\mathrm{X}$-Ray charge which represents here the exposure given in (mAs), mm.Al gives minimum inherent filtration Aluminum equivalent, $\mathrm{kV}_{\mathrm{p}}$ represents $\mathrm{X}$-Ray maximum tube voltage, FSD is focus to skin distance. BSF represents back scattering factor, this factor is roughly equated to one for all investigated examinations. The data obtained was analyzed using professional statistical programmes, which are SigmaPlot and Excel.

The estimated values obtained for the ESD from this research were compared with the international ESD values reported in the literature.

At the beginning the patient's data such as Age and Gender was first recorded and after that the patient was centered by technician at the right position to be prepared for radiographic. The exposure factors such as peak tube voltage $\left(\mathrm{kv}_{\mathrm{p}}\right), \mathrm{X}$-ray charge (mAs) and focus to surface distance (FSD) were recorded at the time of the examination done. This information was recorded for each patient who undergoing the particular diagnostic procedure.

\section{Results}

The research work carried out in Alhilal Alahmar medical center located in Sebha city. One X-ray unit was incorporated in this research work. The results of all obtained data are tabulated below in tables listened from 1 to 6 . The results of this work presented in these tables including: gender, patient's age, peak tube voltage $\left(\mathrm{kv}_{\mathrm{p}}\right)$, exposure factor $(\mathrm{Q})$, focus to surface distance (FSD), examination type, field size, exposure occurrence (this factor gives the number of X-Ray images that taken for each patient at the same diagnostic examination) and entrance-surface-dose (ESD) for all different medical cases being exposed to diagnostic X-rays respectively. The results also included some information related to medical procedures which involving different positions of patients: such as Anterior-Posterior (AP) examination and Lateral (LAT) examination. The other medical procedure included is Posterior-Anterior (PA).The mixed procedures are also considered in this research. In this research, the focus was on six human body parts those exposed to X-rays. These parts include: (i) Lumpier Spine, (ii) Cervical Spine, (iii) Chest, (iv) Foot, (v) Knee, (vi) PNS.

Table 7 indicates the mean values of patient's exposure factors such as $\mathrm{kv}_{\mathrm{p}}, \mathrm{Q}, \mathrm{FSD}$ and ESD and the compression with other studies of the estimated ESD and the established international reference levels of ESD that reported in the literature. 


\section{Clinical Radiology \& Imaging Journal}

\begin{tabular}{|c|c|c|c|c|c|c|c|c|c|}
\hline No. & Gender & Age & KVp (KeV) & $Q(m A s)$ & FSD $(\mathrm{cm})$ & $\begin{array}{c}\text { Examination } \\
\text { Type }\end{array}$ & $\begin{array}{l}\text { Field Size } \\
\text { (cm2) }\end{array}$ & ESD (mGy) & Occurrence \\
\hline 1 & $\mathrm{~F}$ & 39 & 85 & 160 & 90 & \multirow{17}{*}{ LAT } & $45^{\prime} 45$ & 15.84 & 8 \\
\hline 2 & $\mathrm{~F}$ & 65 & 85 & 160 & 85 & & $45^{\prime} 45$ & 17.76 & 7 \\
\hline 3 & $\mathrm{M}$ & 40 & 85 & 120 & 80 & & $45^{\prime} 45$ & 15.04 & 7 \\
\hline 4 & $\mathrm{~F}$ & 55 & 85 & 160 & 70 & & $45^{\prime} 45$ & 26.19 & 6 \\
\hline 5 & $\mathrm{~F}$ & 28 & 85 & 120 & 75 & & $45^{\prime} 45$ & 17.11 & 8 \\
\hline 6 & $\mathrm{~F}$ & 48 & 86 & 160 & 70 & & $45^{\prime} 45$ & 26.6 & 8 \\
\hline 7 & M & 49 & 85 & 160 & 75 & & $45^{\prime} 45$ & 22.81 & 7 \\
\hline 8 & $\mathrm{~F}$ & 26 & 85 & 160 & 80 & & $45^{\prime} 45$ & 20.05 & 4 \\
\hline 9 & $\mathrm{~F}$ & 37 & 84 & 96 & 65 & & $45^{\prime} 45$ & 17.8 & 8 \\
\hline 10 & $\mathrm{M}$ & 72 & 84 & 96 & 70 & & $45^{\prime} 45$ & 15.34 & 6 \\
\hline 11 & $\mathrm{~F}$ & 29 & 83 & 120 & 60 & & $45^{\prime} 45$ & 25.49 & 8 \\
\hline 12 & $\mathrm{~F}$ & 48 & 83 & 120 & 60 & & $45^{\prime} 45$ & 25.49 & 8 \\
\hline 13 & $\mathrm{~F}$ & 31 & 86 & 200 & 70 & & $45^{\prime} 45$ & 33.51 & 7 \\
\hline 14 & $\mathrm{M}$ & 60 & 85 & 120 & 75 & & $45^{\prime} 45$ & 17.11 & 6 \\
\hline 15 & $\mathrm{~F}$ & 72 & 85 & 200 & 65 & & $45^{\prime} 45$ & 37.96 & 7 \\
\hline 16 & $\mathrm{~F}$ & 65 & 86 & 200 & 70 & & $45^{\prime} 45$ & 33.51 & 5 \\
\hline 17 & $\mathrm{~F}$ & 38 & 87 & 120 & 60 & & $45^{\prime} 45$ & 28 & 7 \\
\hline
\end{tabular}

Table 1: Estimated ESD for Lumpier Spine.

\begin{tabular}{|c|c|c|c|c|c|c|c|c|c|}
\hline No. & Gender & Age & $\begin{array}{c}\mathrm{KVp} \\
(\mathrm{KeV})\end{array}$ & $\begin{array}{c}Q \\
(m A s)\end{array}$ & $\begin{array}{l}\text { FSD } \\
(\mathrm{cm})\end{array}$ & $\begin{array}{c}\text { Examination } \\
\text { Type }\end{array}$ & $\begin{array}{l}\text { Field Size } \\
(\mathrm{cm} 2)\end{array}$ & $\begin{array}{c}\text { ESD } \\
(\mathrm{mGy})\end{array}$ & Occurrence \\
\hline 1 & $\mathrm{~F}$ & 29 & 82 & 48 & 100 & \multirow{8}{*}{ LAT } & $45^{\prime} 45$ & 3.58 & 1 \\
\hline 2 & $\mathrm{~F}$ & 38 & 80 & 48 & 100 & & $45^{\prime} 45$ & 3.41 & 1 \\
\hline 3 & $\mathrm{~F}$ & 28 & 82 & 51 & 100 & & $45^{\prime} 45$ & 3.8 & 1 \\
\hline 4 & $\mathrm{M}$ & 38 & 82 & 51 & 100 & & $45^{\prime} 45$ & 3.8 & 1 \\
\hline 5 & $\mathrm{~F}$ & 65 & 83 & 48 & 100 & & $45^{\prime} 45$ & 3.67 & 1 \\
\hline 6 & M & 52 & 83 & 48 & 100 & & $45^{\prime} 45$ & 3.67 & 1 \\
\hline 7 & $\mathrm{~F}$ & 43 & 83 & 48 & 100 & & $45^{\prime} 45$ & 3.67 & 1 \\
\hline 8 & $\mathrm{M}$ & 90 & 84 & 48 & 100 & & $45^{\prime} 45$ & 3.76 & 1 \\
\hline 9 & $\mathrm{~F}$ & 52 & 83 & 54 & 100 & \multirow{2}{*}{$\mathrm{AP}$} & $45^{\prime} 45$ & 4.13 & 1 \\
\hline 10 & $\mathrm{~F}$ & 23 & 83 & 84 & 100 & & $45^{\prime} 45$ & 6.42 & 1 \\
\hline 11 & $\mathrm{~F}$ & 33 & 84 & 48 & 100 & \multirow{4}{*}{ LAT } & $45^{\prime} 45$ & 3.76 & 1 \\
\hline 12 & $\mathrm{~F}$ & 46 & 83 & 83 & 100 & & $45^{\prime} 45$ & 6.34 & 1 \\
\hline 13 & M & 15 & 80 & 80 & 100 & & $45^{\prime} 45$ & 5.68 & 1 \\
\hline 14 & $\mathrm{~F}$ & 38 & 84 & 84 & 100 & & $45^{\prime} 45$ & 6.58 & 1 \\
\hline 15 & $\mathrm{~F}$ & 38 & 38 & 84 & 100 & AP & $45^{\prime} 45$ & 1.34 & 1 \\
\hline
\end{tabular}

Table 2: Estimated ESD for Cervical Spine. 


\section{Clinical Radiology \& Imaging Journal}

\begin{tabular}{|c|c|c|c|c|c|c|c|c|c|}
\hline No. & Gender & Age & $\begin{array}{c}\mathrm{KVp} \\
(\mathrm{KeV})\end{array}$ & $Q(m A s)$ & $\begin{array}{l}\text { FSD } \\
(\mathrm{cm})\end{array}$ & $\begin{array}{c}\text { Examination } \\
\text { Type }\end{array}$ & $\begin{array}{c}\text { Field Size } \\
(\mathrm{cm} 2)\end{array}$ & $\begin{array}{c}\text { ESD } \\
(\mathrm{mGy})\end{array}$ & Occurrence \\
\hline 1 & $\mathrm{~F}$ & 47 & 85 & 48 & 100 & \multirow{17}{*}{ PA } & $45^{\prime} 45$ & 3.8 & 1 \\
\hline 2 & $\mathrm{~F}$ & 34 & 79 & 48 & 100 & & $45^{\prime} 45$ & 3.32 & 1 \\
\hline 3 & $\mathrm{~F}$ & 47 & 85 & 48 & 100 & & $45^{\prime} 45$ & 3.85 & 1 \\
\hline 4 & $\mathrm{M}$ & 17 & 83 & 48 & 100 & & $45^{\prime} 45$ & 3.67 & 1 \\
\hline 5 & $\mathrm{M}$ & 62 & 86 & 48 & 100 & & $45^{\prime} 45$ & 3.94 & 1 \\
\hline 6 & $\mathrm{~F}$ & 55 & 85 & 48 & 100 & & $45^{\prime} 45$ & 3.85 & 1 \\
\hline 7 & $\mathrm{M}$ & 48 & 85 & 48 & 100 & & $45^{\prime} 45$ & 3.85 & 1 \\
\hline 8 & $\mathrm{M}$ & 16 & 85 & 48 & 100 & & $45^{\prime} 45$ & 3.85 & 1 \\
\hline 9 & $\mathrm{~F}$ & 45 & 85 & 48 & 100 & & $45^{\prime} 45$ & 3.85 & 1 \\
\hline 10 & $\mathrm{~F}$ & 50 & 84 & 48 & 100 & & $45^{\prime} 45$ & 3.76 & 1 \\
\hline 11 & $\mathrm{~F}$ & 40 & 84 & 51 & 100 & & $45^{\prime} 45$ & 3.99 & 1 \\
\hline 12 & $\mathrm{M}$ & 20 & 75 & 48 & 100 & & $45^{\prime} 45$ & 2.99 & 1 \\
\hline 13 & $\mathrm{~F}$ & 26 & 84 & 54 & 100 & & $45^{\prime} 45$ & 4.23 & 1 \\
\hline 14 & $\mathrm{M}$ & 26 & 77 & 48 & 100 & & $45^{\prime} 45$ & 3.16 & 1 \\
\hline 15 & $\mathrm{M}$ & 40 & 82 & 48 & 100 & & $45^{\prime} 45$ & 3.58 & 1 \\
\hline 16 & $\mathrm{M}$ & 36 & 85 & 51 & 100 & & $45^{\prime} 45$ & 4.09 & 1 \\
\hline 17 & $\mathrm{M}$ & 52 & 80 & 51 & 100 & & $45^{\prime} 45$ & 3.62 & 1 \\
\hline
\end{tabular}

Table 3: Estimated ESD for Chest.

\begin{tabular}{|c|c|c|c|c|c|c|c|c|c|}
\hline No. & Gender & Age & $\begin{array}{c}\text { KVp } \\
(\mathrm{KeV})\end{array}$ & $\mathrm{Q}$ (mAs) & $\begin{array}{l}\text { FSD } \\
(\mathrm{cm})\end{array}$ & $\begin{array}{c}\text { Examination } \\
\text { Type } \\
\end{array}$ & $\begin{array}{c}\text { Field Size } \\
(\mathrm{cm} 2)\end{array}$ & $\begin{array}{c}\text { ESD } \\
(\mathrm{mGy})\end{array}$ & Occurrence \\
\hline 1 & M & 48 & 54 & 25 & 45 & \multirow{3}{*}{ LAT } & $45^{\prime} 45$ & 3.99 & 1 \\
\hline 2 & $\mathrm{~F}$ & 43 & 49 & 25 & 85 & & $45^{\prime} 45$ & 0.92 & 1 \\
\hline 3 & $F$ & 13 & 48 & 25 & 85 & & $45^{\prime} 45$ & 0.88 & 1 \\
\hline 4 & $\mathrm{~F}$ & 74 & 50 & 25 & 70 & $\mathrm{AP}$ & $45^{\prime} 45$ & 1.41 & 1 \\
\hline 5 & $\mathrm{~F}$ & 74 & 50 & 25 & 65 & \multirow{7}{*}{ LAT } & $45^{\prime} 45$ & 1.64 & 1 \\
\hline 6 & M & 35 & 50 & 25 & 75 & & $45^{\prime} 45$ & 1.23 & 1 \\
\hline 7 & $\mathrm{~F}$ & 52 & 58 & 64 & 90 & & $45^{\prime} 45$ & 2.95 & 1 \\
\hline 8 & $\mathrm{~F}$ & 43 & 57 & 49 & 49 & & $45^{\prime} 45$ & 7.36 & 1 \\
\hline 9 & $\mathrm{~F}$ & 43 & 58 & 64 & 49 & & $45^{\prime} 45$ & 9.95 & 1 \\
\hline 10 & $\mathrm{~F}$ & 50 & 55 & 64 & 49 & & $45^{\prime} 45$ & 8.95 & 1 \\
\hline 11 & $\mathrm{M}$ & 79 & 58 & 49 & 50 & & $45^{\prime} 45$ & 7.32 & 1 \\
\hline
\end{tabular}

Table 4: Estimated ESD for foot.

\begin{tabular}{|c|c|c|c|c|c|c|c|c|c|}
\hline No. & Gender & Age & $\begin{array}{c}\text { KVp } \\
\mathbf{( K e V})\end{array}$ & $\mathbf{Q}(\mathbf{m A s})$ & $\begin{array}{c}\text { FSD } \\
\text { (cm) }\end{array}$ & $\begin{array}{c}\text { Examination } \\
\text { Type }\end{array}$ & $\begin{array}{c}\text { Field Size } \\
\text { (cm2) }\end{array}$ & $\begin{array}{c}\text { ESD } \\
\text { (mGy) }\end{array}$ & Occurrence \\
\hline 1 & F & 30 & 60 & 64 & 25 & AP LAT & $45^{\prime} 45$ & 40.92 & 2 \\
\hline 2 & F & 24 & 60 & 64 & 25 & AP LAT & $45^{\prime} 45$ & 40.92 & 2 \\
\hline 3 & M & 12 & 59 & 64 & 65 & AP LAT & $45^{\prime} 45$ & 5.85 & 2 \\
\hline 4 & M & 45 & 61 & 64 & 70 & AP LAT & $45^{\prime} 45$ & 5.39 & 2 \\
\hline 5 & M & 55 & 62 & 64 & 70 & AP LAT & $45^{\prime} 45$ & 5.57 & 2 \\
\hline 6 & F & 7 & 57 & 49 & 60 & AP LAT & $45^{\prime} 45$ & 4.91 & 2 \\
\hline
\end{tabular}


Clinical Radiology \& Imaging Journal

\begin{tabular}{|c|c|c|c|c|c|c|c|c|c|}
\hline No. & Gender & Age & $\begin{array}{c}\text { KVp } \\
\mathbf{( K e V )}\end{array}$ & $\mathbf{Q}$ (mAs) & $\begin{array}{c}\text { FSD } \\
\mathbf{( c m )}\end{array}$ & $\begin{array}{c}\text { Examination } \\
\text { Type }\end{array}$ & $\begin{array}{c}\text { Field Size } \\
\text { (cm2) }\end{array}$ & $\begin{array}{c}\text { ESD } \\
\text { (mGy) }\end{array}$ & Occurrence \\
\hline 7 & F & 63 & 63 & 10 & 65 & AP LAT & $45^{\prime} 45$ & 1.04 & 2 \\
\hline 8 & F & 65 & 59 & 72 & 60 & AP LAT & $45^{\prime} 45$ & 7.72 & 2 \\
\hline 9 & M & 48 & 59 & 64 & 60 & AP LAT & $45^{\prime} 45$ & 6.87 & 2 \\
\hline 10 & F & 40 & 59 & 64 & 70 & AP LAT & $45^{\prime} 45$ & 5.04 & 2 \\
\hline 11 & F & 32 & 62 & 72 & 65 & AP LAT & $45^{\prime} 45$ & 7.27 & 2 \\
\hline
\end{tabular}

Table 5: Estimated ESD for Knee.

\begin{tabular}{|c|c|c|c|c|c|c|c|c|c|}
\hline No. & Gender & Age & $\begin{array}{c}\text { KVp } \\
\mathbf{( K e V )}\end{array}$ & $\mathbf{Q}(\mathbf{m A s})$ & $\begin{array}{c}\text { FSD } \\
\mathbf{( c m})\end{array}$ & $\begin{array}{c}\text { Examination } \\
\text { Type }\end{array}$ & $\begin{array}{c}\text { Field Size } \\
\text { (cm2) }\end{array}$ & $\begin{array}{c}\text { ESD } \\
\text { (mGy) }\end{array}$ & Occurrence \\
\hline 1 & F & 20 & 85 & 48 & 100 & AP & $45^{\prime} 45$ & 3.85 & 2 \\
\hline 2 & F & 6 & 84 & 48 & 100 & LAT & $45^{\prime} 45$ & 3.76 & 1 \\
\hline 3 & M & $8 \mathrm{~m}^{*}$ & 77 & 45 & 100 & LAT & $45^{\prime} 45$ & 2.96 & 1 \\
\hline 4 & F & 13 & 85 & 48 & 100 & AP & $45^{\prime} 45$ & 3.85 & 1 \\
\hline 5 & F & 48 & 84 & 48 & 100 & PA & $45^{\prime} 45$ & 3.76 & 4 \\
\hline 6 & F & 40 & 84 & 51 & 100 & PA & $45^{\prime} 45$ & 3.99 & 3 \\
\hline 7 & F & 38 & 83 & 54 & 100 & PA & $45^{\prime} 45$ & 4.13 & 6 \\
\hline 8 & F & 28 & 83 & 48 & 100 & PA & $45^{\prime} 45$ & 3.67 & 6 \\
\hline 9 & F & 42 & 84 & 51 & 100 & PA & $45^{\prime} 45$ & 3.99 & 5 \\
\hline 10 & F & 3 & 80 & 48 & 100 & LAT & $45^{\prime} 45$ & 3.41 & 2 \\
\hline 11 & F & 2 & 82 & 48 & 100 & LAT & $45^{\prime} 45$ & 3.58 & 1 \\
\hline 12 & M & 14 & 87 & 51 & 100 & PA & $45^{\prime} 45$ & 4.28 & 4 \\
\hline 13 & F & 2 & 87 & 51 & 100 & AP & $45^{\prime} 45$ & 4.28 & 1 \\
\hline 14 & F & 10 & 80 & 51 & 100 & AP & $45^{\prime} 45$ & 3.62 & 1 \\
\hline 15 & F & 3 & 80 & 48 & 100 & LAT & $45^{\prime} 45$ & 3.41 & 2 \\
\hline 16 & M & 43 & 85 & 51 & 100 & PA & $45^{\prime} 45$ & 4.09 & 4 \\
\hline 17 & F & 31 & 82 & 51 & 100 & PA & $45^{\prime} 45$ & 3.8 & 1 \\
\hline
\end{tabular}

Table 6: Estimated ESD for PNS.

$\mathrm{m}^{*}$ : months

\begin{tabular}{|c|c|c|c|c|c|c|c|}
\hline Diag. Type & $\begin{array}{c}\text { ESD (mGy) This } \\
\text { Study }\end{array}$ & $\begin{array}{c}\text { ESD (mGy) } \\
{[15,16]}\end{array}$ & $\begin{array}{c}\text { ESD (mGy) } \\
{[17]}\end{array}$ & $\begin{array}{c}\text { ESD (mGy) } \\
{[18]}\end{array}$ & $\begin{array}{c}\text { ESD (mGy) } \\
\text { [19] }\end{array}$ & $\begin{array}{c}\text { ESD (mGy) } \\
{[20]}\end{array}$ & $\begin{array}{c}\text { ESD (mGy) } \\
{[21]}\end{array}$ \\
\hline Lumpier Spine (LAT) & 23.27 & 103.7 & 22.61 & 2.25 & 10 & 6 & 10 \\
\hline Cervical Spine (LAT) & 4.31 & 7.25 & 2.4 & $\star$ & * & * & $\star$ \\
\hline Chest (PA) & 3.73 & 7.43 & 0.18 & * & * & $\star$ & $\star$ \\
\hline Foot & 4.23 & * & $\star$ & * & * & $\star$ & $\star$ \\
\hline Knee & 11.95 & $\star$ & * & * & * & $\star$ & $\star$ \\
\hline PNS & 3.79 & $\star$ & * & $\star$ & * & * & $\star$ \\
\hline
\end{tabular}

Table 7: Comparison and Mean Values for Estimated ESDs of Different Examinations.

\section{Discussion}

The aim of this research was strived to evaluate the entrance surface dose (ESD) for patients who go through diagnostic examinations by X-rays. This study was conducted at Alhilal Alahmar medical center. The number of patients included in this study was 87 patients. As it's show from the tabulated data presented above, the protocol of patients' 


\section{Clinical Radiology \& Imaging Journal}

exposure at Alhilal Alahmar medical center is higher $\mathrm{Kv}_{\mathrm{p}}$ lower mAs for Cervical Spine (LAT/AP), Chest (PA), Knee (AP/ LAT) and PNS (AP/PA/LAT). The Lumpier Spine examination uses a different protocol which is higher $\mathrm{mAs}$ lower $\mathrm{Kv}_{\mathrm{P}}$.

The mean values of estimated ESD for Lumpier Spine, Cervical Spine, Chest, Knee and PNS are 23.27 (mGy), 4.31 (mGy), 3.73 (mGy), 4.23 (mGy), 11.95 (mGy) and 3.79 (mGy) respectively. When these obtained values of ESD compared with previous studies conducted in this city [16,17] and international reference levels [18-21] the findings are that: for Lumpier Spine the estimated ESD is a little bit higher than the international reference level. In comparing this examination with other diagnostic examinations we found that the occurrences of each examination occurring from 4 to 8 times per patient. This procedure is definitely maximizing the ESDs for patients experienced this examination.

For Cervical Spine the estimated ESD is within the acceptable range. While for chest the estimated ESD is also within the acceptable range. The minimum ESD value estimated in this study was for chest (PA) while the maximum ESD estimated is for knee.

Based on this discussion we conclude some points: Firstly, most technicians and medical officers still need to be competent in using X-ray machines at radiographic centers. This point is deduced from the surveys performed at this medical center for few mounts during research work. Secondly, all X-ray tubes should undergo calibration tests frequently to minimize higher exposures for patients examined by these diagnostic X-rays.

\section{Acknowledgement}

The researcher would extend his great acknowledgement to technician's staff working at Radiology department in Alhilal Alahmar Medical Center.

\section{References}

1. Ciraj O, Markovic S, Kosutic D (2003) Patient Dosimetry in Diagnostic Radiology. Nuclear Technology and Radiation Protection.

2. (1991) 1990 Recommendations of the International Commission on Radiological Protection, ICRP Publication 60, Ann ICRP.

3. Ciraj O, Markovic S, Kosutic D (2004) Patient Dose from Conventional Diagnostic Radiology Procedures in Serbia and Montenegro. The Journal of Preventive Medicine 12(3-4): 26-34.

4. Alghoul A, Yasir M (2016) Alternative Mathematical
Form for Determining the Effectiveness of High-LET Radiations at Lower Doses Region. Int J Radiol Imaging Technol 2(1): 009.

5. (2000) Avoidance of Radiation Injuries from Medical Interventional Procedures, ICRP Publication 85, Ann ICRP.

6. WHO (1982) Quality Assurance in Diagnostic Radiology. World Health Organization.

7. (1985) CEC, Radiation Protection 33 EUR 9728EN; Office for Official of the EURO Pean Community, Luxembourg.

8. Aliyu A, Mary Morris, Emmanuel D Langa, Garba Ibrahim, et al. (2021) Assessment of Radiation Dose for Patients during X-ray Procedures in University of Maiduguri Teaching Hospital. Journal of Radiation and Nuclear Applications 6(2): 163-169.

9. (1994) Code of Safe Practice for the Use of X-Rays in Medical Diagnosis. National Radiation Laboratory, Ministry of Health.

10. International Commission on Radiation Units and Measurements (1970) Radiation Dosimetry: X-rays Generated at Potentials of 5 to $150 \mathrm{kV}$. Tech Rep 17, Jr. ICRU 2(15).

11. Jornet N, Muñoz J, Martin Viera J, Jurado D, Pallerol R, et al. (2013) Determination of Entrance Surface Dose in Standard Explorations in Radiodiagnostic. New York.

12. Compagnome G, Pagan L, Bergamini C (2005) Comparison of Six Phantoms for Entrance Skin Dose Evaluation in 11 Standard X-ray Examinations. J Appl Clin Med Phys 6(1): 101-113.

13. Shabon M (2013) Pediatric X-ray Examinations (Special Review). Journal of Nuclear and Radiation Physics 82: $35-44$.

14. Alghoul A (2017) Mathematical Evaluation of Entrance Surface Dose (ESD) for Patients Examined by Diagnostic X-Rays. Open Access J Sci 1(1): 00003.

15. Ding C, Wang JP, Yu XR, He SS, Yu FH, et al. (2001) Medical Radiation Usage and Exposures from Medical Radiation X-ray. Diagnostic in Shandong Province of China. Radiat Prot Dosimetry 93(2): 261-266.

16. Chuan J, Tsai H (1999) Evaluation of Gonad Dose and fetal doses for Diagnostic Radiology. Proc Natl Sci Counc Repub China B 23(3): 107-113.

17. Yousif A, Suasey H A, Ramdan M M (2016) Estimation of Entrance Surface Dose (ESD) of Patients Examined 


\section{Clinical Radiology \& Imaging Journal}

by Diagnostic X-Ray at Sebha Medical Center. Journal of Applied Science 16(1).

18. Tamboul J, Yousef M, Mokhtar K, Alfaki A, Sulieman A (2014) Assessment of Entrance Surface Dose for the Patients from Common Radiology Examinations in Sudan. Life Science Journal 11(2): 1-5.

19. (1996) Diagnostic Reference Levels in Medical Imaging, review and additional Advice. a web module produced by committee 3 of the international commission on radiological protection (ICRP).

20. Hull \& East Yorkshire. Hospitals Radiation Protection Service (2005) (Reference Value Proposed as DRL).

21. (1996) Quality Criteria Reference Doses [EUR96], NA. 